\title{
Editorial Desk
}

\author{
Tasneem Ravati* and Geoffrey P Webb \\ SVT College of Home Science, The University of Mumbai, India.
}

Submission: October 04, 2016; Published: October 07, 2016

*Corresponding author: Tasneem Ravat, SVT College of Home Science, The University of Mumbai, India, Email: tasneem_05@yahoo.co.in

\section{Editorial}

Food has a host of cultural, psychological and social functions in addition to its biological function. It is these non nutritional uses that have traditionally been the dominant influences upon our food selection, If we lose sight of these functions in the perhaps fruitless pursuit of the optimal chemical diet, any technical improvements in our nutrition may be outweighed, or at least reduced, by damage to the general emotional health of the population. Miserable, anxious people will probably suffer and die younger than happy, contented people. We have waged a partly successful war against hunger and malnutrition. There has been a slow but steady reduction in under nutrition rates over the past few decades. On the other hand, over nutrition has been emerging as a public health problem in more recent times. Today, while the developing countries are yet to overcome under nutrition and communicable diseases, the rates of over nutrition, obesity and non communicable diseases are climbing.

We are facing this problem of over nutrition because of sharp reduction in physical activity brought about by increasing automation, urbanization and transport infrastructure, as well as a growing trend towards the consumption of 'convenience foods which are often energy dense. These trends are seen not only among the affluent but among all segments of both urban and rural populations. Growing concern over the increase in over nutrition rates stems from the fact that obesity is one of the major risk factors for non communicable diseases. Asians have a higher adiposity for a given BMI as compared to Caucasians, right from birth through childhood and adolescence, and into adulthood. They are more prone to abdominal adiposity and metabolic syndrome. Rising over nutrition rates can substantially accelerate the rate of increase in non communicable disease during the nutrition transition.
This mismatch between energy intake and energy requirement cutting across socio- economic segments, represents an enormous challenge to dietitians. It is clear that the earlier labeling of chronic diseases as "diseases of affluence" is increasingly a misnomer, as they emerge both in poorer countries and in the poorer population groups in richer countries. This rapid rate of change, together with the increasing burden of disease, is creating a major public health threat which demands immediate and effective action.

With lifestyle disorders forcing more and more people to reel under excess body weight, even relatively younger people are developing joint disorders and knee pain. Excessive weight is associated with a series of health problems, including blood pressure, diabetes, and cardiovascular ailments (WHO 2003). Complex diets have shown to impart beneficial results in weight reduction and also in other disease management. It remains possible that particular classes of food or individual foods can confer specific benefit. Sometimes it is difficult to bring about broader dietary changes in the diet in such cases it is preferable to introduce individual diet constituents which may bring about a major change.

Both under and over nutrition, as well as micronutrient deficiencies, can be combated through nutrition and health education and lifestyle counseling. The emphasis should be made on prevention, early detection and effective management of nutrition problems, in close partnership with nutritionists and physicians. There is no service nobler than improving the health and well being of the masses. Our journal publishes research articles which brings new information and is also an interesting read. This is the first issue of our journal and I wish all the readers an enjoyable reading and good health from their everyday food. 


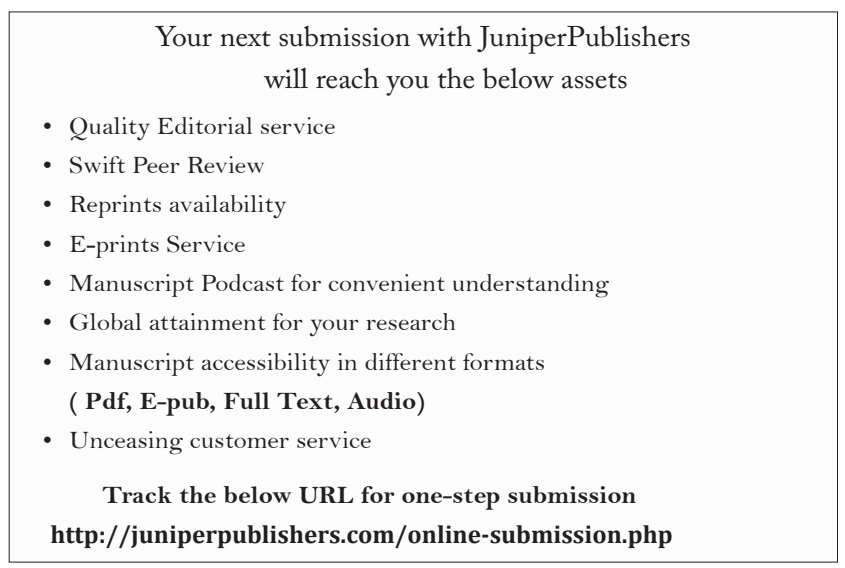

